\title{
El papel del maestro más allá de las estrategias didácticas: apuntes para una propuesta de posicionamiento docente ${ }^{1}$
}

\author{
The teacher's role beyond didactic strategies: \\ Notes for a proposal for teacher positioning
}

\section{Resúmen}

Este documento recoge el análisis de las apreciaciones de docentes en ejercicio sobre su papel como maestros para la sociedad, a partir de interrogantes como: ¿están los docentes llamados a ser simples aplicadores de metodologías?, ¿tienen participación en la creación de propuestas pedagógicas que piensen el tipo de sociedad y de sujeto social que necesita Colombia?, ¿cuál es lugar que se le otorga en países como Colombia a la profesión docente? Para ello, se inicia con un resumen de las competencias docentes que plantean algunos modelos educativos o entidades como la Unesco para reflexionar sobre la visión que se tiene del maestro desde estas entidades. Posteriormente, se exponen las reflexiones de los maestros alrededor de lo que está fallando y qué se debe hacer para erigir al maestro como constructor de tejido social. Se identifican tres tópicos: el lugar del docente en la sociedad colombiana, la formación de profesores y la participación de estos en políticas educativas. Finalmente se presentan conclusiones sobre la importancia del maestro como intelectual transformativo, agente histórico, social, político y cultural, en oposición a la visión instrumentalista del docente como técnico que aplica estrategias didácticas.

Palabras clave: profesión docente, política educativa, competencias docentes, estrategias didácticas, formación docente.

\section{Abstract}

This document gathers the analysis of teachers in practice opinions about their role as teachers for society, based on questions such as: Are teachers called to be simple applicators of methodologies? Do they participate in the creation of pedagogical proposals that think about the type of society and the social subject sought in Colombia? What place is given in countries like Colombia to the teaching profession? For this, an initial summary of the teaching competences that some educational models or entities such as Unesco seeks is offered to generate reflection about the vision such entities have of teachers. Afterwards, teacher's reflections about what is failing are exposed and what must be done to position teachers as social fabric builders, identifying three topics: the teacher's place in Colombian society, teacher's training, and their participation in educational policies. All of these to finally reach conclusions about the importance of teacher's as transformative intellectuals, historical, social, political and cultural agents, in opposition to the instrumentalist vision of teachers as technicians applying didactic strategies.

Keywords: teaching profession, educational policy, teaching skills, teaching strategies, teacher training.

\footnotetext{
1 Artículo de investigación derivado de la tesis doctoral de la licenciada Norma Lucía Muñoz, financiado con recursos propios. 2 Licenciada en Biología y Química de la Universidad del Valle. MSc. en Ingeniería Sanitaría y Ambiental de la Universidad del Valle, estudiante de doctorado interinstitucional en Educación (Universidad del Valle, Universidad Pedagógica Nacional, Universidad Distrital Francisco José de Caldas); línea de educación en ciencias, Universidad del Valle, Cali, Colombia. Correo electrónico: norma.munoz@correounivalle.edu.co
} 


\section{Introducción}

Los docentes, sin lugar a dudas, tienen un papel fundamental en todas las propuestas que se realizan a nivel educativo, son ellos quienes finalmente movilizan esas nuevas miradas de los procesos de enseñanza, aprendizaje y evaluación en el aula. Por ello, parte importante del éxito de los modelos propuestos para la educación pasa por el convencimiento y conformidad que los docentes tengan sobre dichos modelos, así como por su profesionalismo y compromiso para hacerlos realidad en la cotidianidad escolar.

Así pues, cada propuesta pedagógica o didáctica requiere una serie de características que el docente debe comportar y/o desarrollar en su quehacer profesional para lograr el éxito de la misma. Es así que, dadas las características atribuibles a los docentes, se puede elaborar una larga lista de competencias que van desde el conocimiento disciplinar y didáctico, pasan por los modelos pedagógicos, el desarrollo de capacidades relacionadas con el manejo de emociones, la responsabilidad social y la administración, por mencionar lo general solamente. Ante lo cual, bien valdría la pena preguntarse si la tarea del docente es la de alcanzar las competencias dispuestas en esos modelos y ser un simple aplicador de las propuestas realizadas por los "expertos" o si puede (o está llamado a) pensarse el tipo de ciudadano que quiere coadyuvar a formar, en concordancia con la sociedad en la que quiere vivir.

Se vive un momento crucial para la educación colombiana, en el cual se debe pensar en la formación de ciudadanos preparados para asumir los retos que presenta la sociedad actual y esto indiscutiblemente pasa por la razón de ser de la escuela, la educación y el quehacer docente; por ello es urgente generar una discusión seria sobre estos temas que logre posicionar la mirada académica en el discurso político que pretende aceptación en los organismos internacionales para estandarizar métodos y que desconoce las realidades específicas y diversas de nuestro país.
Con este escrito se espera contribuir a dicha discusión en cuanto se refiere a la realidad vivida por el profesorado colombiano y sus propósitos y expectativas, puesto que es innegable que el docente es el actor principal del funcionamiento del sistema educativo de una nación.

\section{Metodología}

Inicialmente, se hace una revisión analítica sobre las competencias que deben tener los docentes -según diversas propuestas educativas- para posteriormente discutir si estas competencias docentes "deseadas" se pueden hacer realidad en el ejercicio de la práctica pedagógica o son propuestas utópicas en contextos como los nuestros.

Con el objetivo de continuar con la discusión sobre la función de la labor docente con un punto de vista más amplio, se entrevistó a 107 docentes en ejercicio vinculados a la educación básica y media de 45 instituciones educativas oficiales de la ciudad de Cali, sin discriminar su área de desempeño.

La entrevista semiestructurada aplicada contó con las siguientes preguntas:

- ¿Están los docentes llamados a ser los aplicadores de las metodologías pensadas por los funcionarios del Estado contratados para esta tarea?

- ¿Tienen los docentes participación en la creación de propuestas pedagógicas que piensen el tipo de sociedad y de sujeto social que necesita Colombia?

- ¿Cuál es el lugar que se le otorga en Colombia a la profesión docente?

- ¿Existen condiciones económicas, sociales, políticas para que sea posible una formación de docentes que apunte a desarrollar las competencias deseadas durante la formación y el servicio? 
Posteriormente, se analizaron los resultados de las entrevistas con el software para análisis de datos cualitativos Atlas.ti que permitió organizar la información y facilitar su posterior estudio; de esta manera, las respuestas, reflexiones y aportes de los maestros se clasificaron bajo tres aspectos: el lugar del profesor en la sociedad, la formación docente y la participación de profesores con experiencia en el ejercicio docente en el planteamiento de la política educativa del país. Finalmente, se elaboraron las conclusiones.

\section{Revisión analítica}

\section{Competencias docentes según diversas propuestas educativas}

Como ya se ha mencionado, los profesores son uno de los ejes fundamentales en la dinámica escolar y en la cotidianidad con que esta pretende formar cierto tipo de ciudadano; en ellos recae la responsabilidad de llevar a la práctica los diferentes modelos educativos. Es por esto que cada propuesta o modelo educativo plantea un "deber ser" para los docentes, unas competencias que han de desarrollar y llevar al ejercicio cotidiano para obtener los resultados óptimos que pretende cada una de las diversas iniciativas.

Varios autores han planteado formas variadas de clasificación de los docentes a partir de las características que le son otorgadas en los diferentes modelos pedagógicos que practican; para efectos de síntesis, aquí se presentan de manera rápida aquellos propuestos por Ruiz (2007) y Torres (2004). En primer lugar, se puede reconocer el modelo que se centra en la enseñanza por transmisión - recepción en la cual el docente es el portavoz de la ciencia y por lo tanto, debe dominar los saberes propios de su ámbito de enseñanza, tener la capacidad de explicar con rigurosidad, claridad y precisión, apuntar los resultados de la actividad científica, además de escoger de forma cuidadosa las situaciones problémicas que los educandos han de resolver, y que se caracterizan por privilegiar indagaciones de tipo cerrado y cuantitativo.
En segundo lugar se encuentra el modelo que privilegia la enseñanza por descubrimiento. En este, el docente debe desarrollar competencias que le permitan ser coordinador/guía del trabajo en el aula, que busque inducir a los estudiantes a encontrar las respuestas a los problemas planteados y a desarrollar destrezas de investigación: observación, planteamiento de hipótesis, experimentación, etc. Es decir, provoca y facilita aprendizajes al asumir su labor, no en términos de enseñar, sino de lograr que los alumnos aprendan. Además, prepara a los estudiantes para que seleccionen y utilicen críticamente la información proporcionada por los medios de comunicación masivos, en términos de utilidad.

El tercer modelo hace énfasis en lo que se denomina la enseñanza por recepción significativa, en este el docente debe ser fundamentalmente, un guía en el proceso de enseñanza-aprendizaje, para lo cual debe utilizar como herramienta metodológica la explicación y la aplicación de los denominados organizadores previos, empleados como conectores de índole cognitivo entre los presaberes del educando y la nueva información que el docente lleva al aula. Lo anterior requiere que el docente ejerza un criterio profesional para discernir y seleccionar los contenidos y didácticas más adecuados en cada contexto y para cada grupo, lo que a su vez demanda un alto conocimiento sobre los estilos de aprendizaje de los estudiantes.

Hasta aquí se han mencionado competencias docentes necesarias para llevar a cabo algunos modelos educativos que en genérico se han denominado estructurantes (heteroestructurantes o autoestructurantes), pero es claro que la cotidianidad escolar hace que se plantee, desde la teoría, una lista aún más larga de competencias, puesto que los docentes deben interactuar, no solo con la enseñanza como en el pasado, sino con las disciplinas y prácticas derivadas de los saberes específicos; el maestro de hoy en día es animador, facilitador, guía, orientador (Jiménez, 2011). Al respecto, Torres (2004) recoge varias características docentes que pasan, no solamente por la disciplina, la didáctica y la relación 
docente-estudiante-familia, sino que plantean también tareas asistenciales, resultado de la creciente presión sobre la institución escolar para que se haga cargo de los problemas vinculados con la pobreza, las crisis familiares y el desempleo, algunas son:

- Reflexionar críticamente sobre su papel y práctica pedagógica, sistematizar y compartir en espacios de inter-aprendizaje.

- Comprender la cultura y las realidades locales, llevar a cabo una educación bilingüe e intercultural en contextos bi y plurilingües.

- Detectar oportunamente problemas (sociales, afectivos, de salud, de aprendizaje) entre sus alumnos para así derivarlos a quien corresponde o buscar las soluciones en cada caso.

- Interpretar y aplicar un currículo, tener la capacidad para recrearlo y construirlo para responder a las especificidades locales.

- Desarrollar una pedagogía activa basada en el diálogo, la vinculación de teoría y práctica, interdisciplinariedad, diversidad y trabajo en equipo.

- Impulsar actividades educativas que vayan más allá de la institución escolar, incorporar así a los que no están, recuperar a los que se han ido, y atender a necesidades de los padres de familia y la comunidad como un todo.

Cabe destacar las características docentes planteadas por la Organización de las Naciones Unidas para la Educación, la Ciencia y la Cultura (Unesco, por sus siglas en inglés) y la Organización para la Cooperación y el Desarrollo Económicos (OCDE) que refuerzan esas tareas de tipo asistencia social propuestas para la escuela:

- Los alumnos perciben al docente como un amigo y un modelo, como alguien que les escucha y les ayuda en su desarrollo (Unesco, 1996).

- El profesor desarrolla y ayuda a sus alumnos a desarrollar los conocimientos, valores y habilidades necesarios para aprender a conocer, aprender a hacer, a vivir juntos, y a ser-pilares de la educación propuestos por Unesco- (Delors, 1997).

- El docente responde a los deseos de los padres con respecto a los resultados educacionales, a la necesidad social de un acceso más amplio a la educación y a las presiones en pro de una participación más democrática en las escuelas (OCDE, 1991).

La anterior recopilación muestra las expectativas y responsabilidades que descansan sobre los maestros, tanto a nivel personal como profesional. Se destaca que, sin importar cuán diferentes sean las propuestas en el aula, los docentes siempre tienen un papel de suma importancia para su implementación; es así como se observa que en todas las propuestas mencionadas el docente es quien organiza y prepara la actividad, la coordina durante su ejecución y finalmente evalúa. Para ello, debe tener capacidades intelectuales que le permitan interactuar con el conocimiento y tomar decisiones, habilidades de manejo de grupo que le faculten para llevar las situaciones a buen término, competencias personales que le motiven y le garanticen llegar a los estudiantes de la manera en que desea y sentido social que le permita promover y garantizar en la escuela aquellos derechos que por fuera de ella se ven vulnerados.

Se podrían continuar enumerando las características docentes descritas en las diversas propuestas sobre el papel del profesor, pero es necesario hacer un alto y ver más allá de las competencias docentes que muestran a los profesionales de la educación como simples aplicadores de estrategias pedagógicas o de políticas de Estado e indagar sobre las posibilidades de incluirlos como intelectuales que piensan modelos (no solo educativos), el papel de los sujetos en ellos y la sociedad que se está reproduciendo desde la escuela. Para ello, es indispensable abrir la discusión al respecto teniendo en cuenta, cuando menos, algunos puntos neurálgicos como los que se plantean a continuación. 


\section{Competencias docentes "deseadas": realidad o utopía}

Aunque resulta comprensible que las propuestas para mejorar los procesos de enseñanza, aprendizaje y evaluación planteen una serie de competencias docentes para lograr el propósito trazado, cabe mencionar dos dificultades que estas pueden presentar, la primera es que pueden olvidar los contextos específicos y hacer planteamientos que se podrían volver irreales o imposibles de conseguir en algunos casos; y la segunda es que por lo general, adolecen de estrategias que permitan a los docentes entender cómo desarrollar esas competencias para llevar a buen término la propuesta en el aula de clase.

Así pues, con este panorama en mente resulta fundamental reflexionar sobre las propuestas sobre competencias docentes necesarias para la aplicación de modelos, no únicamente porque sean difíciles de alcanzar o porque se salgan de la dinámica de la escuela y se pretenda suplir la inestabilidad familiar o social, sino porque es indispensable ir más allá e indagar por la intencionalidad de las múltiples competencias docentes deseables que circundan hoy en el medio educativo; como lo plantea Ruiz (2007), los docentes no son técnicos que se limitan a la aplicación de mandatos o instrucciones estructuradas por "expertos" o personas dedicadas a la transmisión de unos conocimientos, son seres humanos con modelos mentales que orientan sus acciones, con unas concepciones o ideas con respecto a su ejercicio profesional que direccionan su quehacer docente y que además, facilitan u obstaculizan el desarrollo de los procesos de enseñanza y aprendizaje.

\section{Resultados y discusiones}

Los planteamientos que se esponen a continuación surgen de las discusiones de profesores en ejercicio de básica y media de diferentes áreas, que fueron entrevistados a partir de las preguntas planteadas en la metodología y que están vinculados a instituciones educativas de carácter oficial de la ciudad de Cali. En general, las reflexiones y aportes de los maestros, luego de ser gestionadas en el software de análisis cualitativo Atlas.ti, se pueden organizar en tres aspectos: el lugar del profesor en la sociedad, la formación docente, y la participación de profesores con experiencia en el aula en el planteamiento de la política educativa del país.

\section{El lugar del profesional docente en la sociedad colombiana}

Para los profesores entrevistados es innegable que, mientras la labor docente sea vista como un oficio sin mayor relevancia social, las licenciaturas seguirán siendo escogidas, en la mayoría de los casos, como última opción y no por vocación, pues son las carreras que piden menores puntajes en las pruebas de admisión y en las universidades privadas son las de menores costos.

Esto se puede observar en la apreciación de un docente cuando manifiesta:

El lugar que le da este país a la profesión docente es mínimo, eso empieza desde el ingreso a la universidad a hacer una licenciatura, prácticamente cualquiera puede entrar a ella por los pocos requerimientos, se puede hacer la comparación con medicina o una ingeniería, son carreras costosas con resultados de las pruebas estatales bastante altos. (entrevista semiestructurada, abril de 2018)

Así, la mirada que en el país predomina sobre los profesores es que son profesionales preparados con poca rigurosidad, cuya labor es comparable con la de cuidadores, maltratados por las políticas estatales. La revista Semana incluso planteó que la educación no está en manos de los mejores porque a las licenciaturas ingresan los bachilleres con peor desempeño en las pruebas de Estado Saber 11 (Semana, 2016). Esta realidad contrasta con los modelos de excelencia educativa del mundo, ejemplo de ello son Finlandia o Shanghái, en donde se exigen los mejores puntajes para ingresar a las facultades de educación. 
La realidad mundial demuestra que los países en los cuales tiene mayor prestigio la profesión docente son también los de mejores resultados en sus estudiantes a nivel internacional ${ }^{3}$, es el caso de China, Corea del sur, Finlandia, Canadá y Singapur, tal como lo expresó Andreas Schleicher, director de educación de la OCDE, "estos países tienen formas muy diferentes de enseñar, pero todos ellos conceden un gran valor a los maestros" (CNN, 2017).

Otro aspecto que resaltan los docentes para indicar el no reconocimiento de la sociedad y el Estado colombiano a su labor son los bajos salarios que se les asigna. A este respecto uno de los docentes apuntó:

La poca importancia que le da este país a la educación y a los docentes se ve reflejada en los pobres salarios para los docentes, mientras otros profesionales estudiando los mismos cinco años tienen salarios más o menos cómodos, los docentes tenemos que estar haciendo paros para que se nos haga una nivelación salarial. (entrevista semiestructurada, abril de 2018)

Aunque esta realidad ha estado cambiando paulatinamente, según el informe de Morduchowicz y Duro (2007), en Colombia para el 2017 los profesionales tenían un ingreso de $\$ 4.494 .373 \mathrm{cop}$, los profesionales y técnicos asalariados de 4.571 .0099 cop y los docentes de 3.789.084 cop. Mientras que para el 2018, luego de observar las tablas salariales del Ministerio de Hacienda y Crédito Público, el salario de los profesionales iba desde $\$ 1.773 .036$ cop hasta $\$ 7.344 .289$ cop, según el grado salarial (Decreto 330 de 2018) y para los docentes iba desde $\$ 1.896 .063$ cop hasta \$7.163.444 cop, claro está, para los docentes regidos por el Decreto 1278 de 2002 (Decreto 316 de 2018).

Sin embargo, es necesario reconocer que este ajuste salariar paulatino se ha dado gracias a las exigencias del magisterio debidas a la presión de las movilizaciones y los paros, lo que hace que socialmente, el profesor sea visto como un profesional golpeado por las políticas estatales.

3 Medición realizada por la prueba PISA (Programa para la Evaluación Internacional de Alumnos), aplicada por la ocde a estudiantes de 15 años en sus países adscritos, en matemáticas, lectura y ciencias, realizada cada tres años.
La pregunta que sigue es entonces: ¿cómo se puede posicionar socialmente la labor docente? Es posible recabar algunas pistas de las experiencias internacionales exitosas, por ejemplo: elegir a sus futuros profesores entre los mejores alumnos que salen de la enseñanza media; educarlos con los mejores profesores universitarios; garantizarles un puesto de trabajo después de la formación; hacer un acompañamiento durante los primeros años de ejercicio de la profesión (Enkvist, 2010). En otras palabras, dar mayor peso a la selección de los futuros maestros y garantizar una formación de calidad, no solo durante el ciclo de profesionalización sino también durante su ejercicio.

Sin duda, un aspecto de suma importancia para mejorar la calidad educativa de un país es la valoración de la figura del profesor, así pues, es necesario que cada docente interiorice la importancia social de tener en sus manos la formación de ciudadanos y que el Estado promueva estrategias para dar estatus a esta labor; como plantea Schleicher, "Los países con el mejor desempeño, tienen algo en común, el estatus de la profesión es consecuencia de que es intelectualmente tentador convertirse en un maestro" (CNN, 2017).

\section{La formación docente}

En la actualidad existe un llamado de los docentes que continúan su formación en maestrías y doctorados para mejorar la formación inicial de los licenciados, pues su experiencia ha sido que la formación recibida durante el pregrado se queda corta para asumir la realidad en el aula. Además, es indispensable profundizar en las estrategias para enseñar a pensar, para reflexionar sobre la labor docente como una actividad de intelectuales que piensan su quehacer, que lo contrastan con experiencias de otros y que generan propuestas en aras de mejorar la calidad educativa del país; esto tanto en el ciclo de formación inicial como en la formación durante el servicio, puesto que es necesario e indispensable que los profesores continúen su 
formación y especialización, aún después de obtener su título profesional.

Al respecto, la opinión general de los profesores entrevistados es que no existen las condiciones económicas, sociales y políticas para que sea posible una formación de docentes que apunte a desarrollar las competencias deseadas durante la formación y en el servicio, ya que esto está sujeto a las posibilidades de cada profesor debido a que el apoyo estatal para su formación postgradual no es una política de Estado sino un proyecto que cada gobierno decide ejecutar, o no y como apunta uno de los docentes:

[...] Cuando un maestro no puede acceder a una beca para un postgrado, debe hacer su propio financiamiento, pero precisamente son los profesores que menos ganan los que aún no tienen una maestría o un doctorado, es decir, financiarlos con recursos propios es muy difícil [...] (entrevista semiestructurada, abril 2017)

Ahora bien, se requieren cambios estructurales que pasan por transformar algunos aspectos de las facultades de educación, no vendría mal retomar casos como los de Finlandia en los que, como ya se mencionó, para ingresar a la formación en docencia se requieren promedios altos y pasar una prueba de selección en la cual se tiene en cuenta la capacidad de comunicación y empatía, un resumen de la lectura de un libro, una explicación de un tema frente a una clase, una demostración de aptitudes artísticas, una prueba de matemáticas y otra de aptitudes tecnológicas (Arrizabalaga, 2012).

En varios lugares, la carrera comprende un periodo de tres años de licenciatura y dos de un magíster de especialización en los cuales la exigencia es bastante alta, pues pretende formar expertos en educación. Los profesores que orientan a estos futuros docentes trabajan sobre experiencias concretas, discuten lo que ha pasado en el aula y basan su ayuda en escuchar, conversar y construir confianza entre el profesor y el joven estudiante en formación. Para el ejercicio de la profesión, la enseñanza infantil selecciona los mejores docentes, debido a que esta etapa se considera la más importante en el proceso educativo. El profesor de los niveles superiores debe estudiar su materia principal durante por lo menos dos años y después estudiar también dos materias más durante por lo menos un año (Enkvist, 2010). La formación de los docentes en ejercicio es también muy importante, en Singapur, por ejemplo, otro país con resultados destacados en educación, los profesores tienen más de 100 horas de desarrollo y formación profesional cada año (CNN, 2017).

Se han mencionado experiencias exitosas en otros lugares del mundo que no es imposibles realizar en Colombia, hay que comenzar a trabajar en los ajustes; no se trata solo de falta de recursos, se trata de su redistribución e inversión en formación que realmente sea de calidad.

\section{Participación en políticas educativas}

Una queja constante de los docentes colombianos es que los lineamientos para la educación primaria, secundaria y media la realizan expertos en pedagogía con un amplio recorrido académico, pero que ignoran la realidad del aula, porque no tienen experiencia en ella.

Por ello, el profesor con experiencia en la práctica docente debe ser tenido en cuenta y convocado al equipo de expertos que definen los lineamientos, metodologías y modelo educativo del país, esto es, el equipo que piensa qué tipo de ciudadano colombiano se quiere formar.

Es fundamental que los profesores dejen de ser los simples aplicadores de modelos, metodologías y estrategias, que asuman su labor desde pensar el modelo educativo para el país y dar así respuesta a cuál es la sociedad que queremos. Esto obviamente, no se logra de la noche a la mañana y con la sola voluntad de los docentes, se requiere preguntar 
por la visión de la educación que tiene el país, el financiamiento asignado para la educación como política de Estado y no como programa de gobierno y el respeto por la profesión docente, entre otros aspectos necesarios para lograr una verdadera transformación del sistema educativo tal que aporte a la formación de ciudadanos comprometidos con el conocimiento, el desarrollo social, ambiental y económico en condiciones de vida digna para todos.

\section{Conclusiones y recomendaciones}

Si bien es importante indagar sobre modelos didácticos y las competencias que dichos modelos requieren de los docentes para llevarlos a cabo -ya que constituyen herramientas fundamentales para el quehacer cotidiano en el aula de clase-, los docentes se deben convencer y asumir un rol de profesional-intelectual de la educación, buscar incidir en las políticas educativas y de esta manera, en la construcción de la sociedad. Como lo plantea McLaren (2003), el maestro se debe concebir como sujeto generador de tejido social y como agente esencialmente cultural, lo que en términos de Giroux (1990) sería un intelectual transformativo.

Para lograr lo anterior resulta fundamental que se reconozca al docente como agente histórico, social, político y cultural que produce conexiones, redes de significado social, simbolizaciones y rutas de sentido (Díaz, 2006), en oposición a la visión instrumentalista del docente como técnico aplicador de estrategias didácticas.

Es hora de actuar sobre los aspectos mencionados en este documento: la invisibilización de los docentes de secundaria y media en la elaboración de las políticas educativas, debido a que no se han establecido protocolos para que la participación de estos profesores sea posible; la formación de los maestros que en Colombia es para los estudiantes con menores puntajes en las pruebas estandarizadas; la capacitación ofrecida por el Ministerio de Educación Nacional para los docentes en ejercicio, que no es de calidad y obedece a cursos masivos de actualización de docentes vinculados a proyectos inmediatos y no a políticas de Estado; y finalmente, el valor social de la labor docente en el país, que es relativamente, de "poca importancia". Un profesor es visto como un profesional con una labor agobiante, y no como un intelectual, investigador, constructor y transformador del entorno político, social, económico, ambiental, que lo rodea.

Se debe intervenir en los procesos de formación docente tanto a nivel profesional como en servicio para hacer del maestro un ser humano en permanente construcción, autónomo, creativo, lector crítico de la realidad, sensible, comprensivo e investigador, abierto a las relaciones interdisciplinarias, ubicado en un mundo ético en el cual se compromete con el otro (Jiménez, 2011) y el cuidado del medioambiente.

Es necesario promover a los docentes como profesionales que se piensen el quehacer cotidiano en el aula con una finalidad específica de construcción de sociedad y de sujeto social para lograr así su vinculación a la esfera política de toma de decisiones y otorgarle voz y voto en la instancia en la que se construye el modelo educativo del país con una intencionalidad concreta de modelo social.

Los docentes están llamados a participar activamente en movimientos pedagógicos que dignifiquen su labor, que coloquen al maestro en el centro de la discusión educativa, tal que les dé elementos de participación, pero también posibilidades de decisión. Uno de los primeros objetivos de este movimiento es que los docentes en sí comprendan la importancia social de su labor y su rol como profesionales, intelectuales, constructores de nación. 


\section{Referencias}

Arrizabalaga, M. (2012, octubre 9). Así consigue Finlandia ser el número 1 en Educación en Europa. Diario ABC [En línea). Recuperado de http://www.juntadeandalucia.es/averroes/ centros-tic/23002255/helvia/sitio/upload/ abciconsiguefinl.pdf

CNN (2017, octubre 29). ¿Dónde están los maestros mejor pagados y valorados del mundo? CNN. Recuperado de https://cnnespanol.cnn. com/2017/10/29/donde-estan-los-maestrosmejor-pagados-y-valorados-del-mundo/ consultado el 11 de julio de 2018

Delors, J., (1997). Informe de la Unesco sobre la educación para el siglo xxi. Santillana Ediciones Unesco

Díaz M., C. J. (2006, enero-junio). Subjetividad docente y resistencias culturales: entre la constricción instrumental y la posibilidad del sentido como creación. Revista Actualidades Pedagógicas, (48), 95-103.

Enkvist I. (2010). El éxito educativo finlandés. Finland's success in PISA. Universidad de Lund (Suecia) [Ponencia en el iv Congreso Iberoamericano de Pedagogía]. Revista Bordón, 62(3), 49-67.

Giroux, H. (1990). Los profesores como intelectuales. Barcelona: Paidós.

Jiménez, J. R. (2011). La subjetividad del maestro en Colombia: La tensión entre la formación institucional y las prácticas de formación (19602002). Revista Educación y Ciudad, (20), 4758. Recuperado de https://revistas.idep.edu.co/ index.php/educacion-y-ciudad/article/view/96
McLaren, P. (2003). La vida en las escuelas. Una introducción a la pedagogía crítica en los fundamentos de la educación. México: Siglo XxI Editores.

Morduchowicz, A., y Duro, L. (2007). La inversión educativa en América Latina y el Caribe. Las demandas de financiamiento y asignación de recursos. Buenos Aires, Argentina: Unesco, Instituto Internacional de Planeamiento para la Educación (IIPE).

OCDE (1991). Escuelas y calidad de la enseñanza (Informe internacional). Barcelona: Paidós, MEC.

Ruiz, F. J. (2007, julio-diciembre). Modelos didácticos para la enseñanza de las ciencias naturales. Revista Latinoamericana de Estudios Educativos, 3(2), 41-60.

Semana (2016, enero 16). ¿Cuáles son los mejores países para ser profesor? Semana. Recuperado de https://www.semana.com/educacion/articulo/ los-mejores-paises-para-ser profesor/456725-3

Torres, R. M. (2004). Nuevo rol docente: ¿qué modelo de formación, para qué modelo educativo? Revista colombiana de educación, (47). https:// doi.org/10.17227/01203916.5512

Unesco (1996). Plan de Escuelas Asociadas, ¿Cómo debe ser un buen maestro? Los niños opinan. Paris: Unesco. 